\title{
An die Ärztinnen und Ärzte in der Grundversorgung
}

Liebe Kolleginnen und Kollegen

Wir stehen mitten im Dezember, haben sechs Monate Erfahrung mit dem TARMED UVG, und in zwei Wochen wird der TARMED KVG in Kraft treten. Wir haben die ersten Erfahrungen gesammelt, den Tarif kriegen wir nach und nach in den Griff, und sein genereller Einsatz für all unsere Konsultationen sollte eigentlich keine übermässigen Probleme bieten.

Nun ist jedoch vor zwei Wochen eine Nachricht eingetroffen, die uns alarmieren sollte: Die durchschnittlichen Kosten pro Unfallbehandlung sind dabei, weit über die fixierten Normen für die Kostenneutralität hinauszuschiessen!

Worum geht es? Es ist nicht sehr kompliziert...

Von politischer Seite war die Vorgabe für den TARMED von Anfang an, dass die Einführung dieses Tarifs auf keinen Fall Anlass zu einer Anhebung der globalen Kosten geben darf; kurz gesagt dürfen somit für die gesamte Ärzteschaft die durchschnittlichen Behandlungskosten pro Fall während der ersten 18 Monate des neuen Tarifs innerhalb einer gewissen Bandbreite nicht höher liegen als in den letzten paar Jahren.

Seit zwei Monaten übersteigen jedoch die von uns Hausärzten ausgestellten Rechnungen diese Limite bei weitem!

Gewiss, es war vorgesehen - und wünschenswert -, dass der TARMED unsere «intellektuellen» Leistungen gegenüber den «technischen» der Spezialisten aufwertet, aber die jetzt feststellbare Entwicklung geht eindeutig zu weit! Wir sind im Begriff, die Kosten derart in die Höhe zu treiben, dass eine Senkung des Punktewerts unvermeidbar zu werden droht.

Um einen solchen Schritt zu vermeiden, der die gesamte Ärzteschaft bestrafen würde, oder um andere Massnahmen zu verhindern, die speziell uns, die Hausärzte, bestrafen würden, haben die Präsidenten der SGAM, der SGIM und der SGP die TARMED-Assessment-Kommission am vergangenen 12. Dezember getroffen, um über die zu ergreifenden Massnahmen zu diskutieren.

Wir werden selbstredend so hart als nötig für die Wahrung unseres Standpunkts kämpfen und uns für die berechtigten Interessen unserer Mitglieder einsetzen.

Doch an Sie alle ergeht ein dringender Appell: Uns bleiben drei Monate, nicht mehr, um den Beweis zu erbringen, dass sich der Taxpunkt von Fr. 1.halten lässt!

Es scheint effektiv ziemlich klare und schlüssige technische Erklärungen für die festgestellte Übermarchung zu geben - Jürg Pellaton erklärt sie auf den folgenden Seiten, und wir bitten Sie, sie mit grösster Aufmerksamkeit zu lesen.

Im Moment scheint es aus Sicht aller Vertragspartner noch durchaus möglich, den Unfalltaxpunkt auf Fr. 1.- zu belassen und mithin die Vorteile, die wir Hausärzte aus dem TARMED ziehen, zu wahren - doch dazu muss sich jede und jeder von uns Allgemeinärzten/-ärztinnen, Internisten/-innen und Kinderärzten/-ärztinnen an eine angemessene Anwendung des Tarifs halten.

Wir danken Ihnen dafür, dass Sie den Tarif entsprechend den von Jürg Pellaton zusammengefassten Richtlinien und Empfehlungen anwenden und damit unsere Glaubwürdigkeit und Reife beweisen.

Jacques de Haller, Präsident SGAM

Werner Bauer, Präsident SGIM

Pierre Klauser, Präsident SGP

Marc Müller, Präsident KHM 